\title{
Solar Rotation over Solar Cycle 21
}

\author{
Elisabeth Ribes ${ }^{1}$, Istvan Vince ${ }^{2}$, Pierre Mein ${ }^{1}$, \\ Eduardo Neto Ferreira ${ }^{1}$
}

${ }^{1}$ URA 326, Observatoire de Paris, 92195 Meudon, France

${ }^{2}$ Osservatorio Beograd, Beograd, Yugoslavia

\begin{abstract}
Having measured the rotation rate of sunspots through solar cycle 21, from 1977 to 1983 , we have found that the mean differential rotation averaged over this seven year record is similar to the grand average differential rotation determined by Howard et al. (1984) over the period 1921-1982. However, the rotation rate does change from year to year. These changes are evidenced by a steepening or a flattening of the mean differential rotation profile, as well as significant changes in the equatorial rate. The presence of a time-dependent pattern of azimuthal rolls inferred from the meridional circulation pattern of the sunspots offers a qualitative explanation of the observed rotation rates. The amplitude of the changes is almost one order of magnitude larger than that of the torsional oscillations found by Howard and LaBonte (1981).
\end{abstract}

\section{Introduction}

The rotation of the solar surface has been thoroughly explored by means of spectroscopic measurements of the photospheric plasma (Howard and LaBonte, 1980), and it has been found that the surface rotation exhibits changes as solar cycle proceeds. The mean differential rotation sometimes steepens and sometimes flattens out. This phenomenon has been described as a "torsional oscillation". Magnetic tracers have also been used, e.g., from measurements of individual sunspot positions on the Mt. Wilson white-light plate collection for the period 1921-1982, grand average rates have been determined that indicate significant changes in the solar rotation (Gilman and Howard, 1984), but which the authors felt were different from the torsional oscillation detected on the spectroscopic measurements.

In order to investigate these changes, we have made use of the Meudon collection of spectroheliograms which exhibit various magnetic features. A digital analysis of the spectroheliograms taken in the violet wing of the chromospheric Ca II line $(393.3 \mathrm{~nm})$ provides a more accurate detection of sunspots than by the conventional methods. 


\section{Methods and results}

In most studies involving sunspots as tracers of the surface rotation, the accuracy of individual sunspot velocity field observations is usually no better than 0.2 per day, i.e. $25 \mathrm{~ms}^{-1}$. Sunspot rotation rates exhibit a considerable scatter, so substantial time and spatial averaging are required to obtain reliable results (Howard et al., 1984; Balthasar et al., 1986). If the changes in rotation rate are of solar origin and time-dependent, it is important to locate sunspot positions with greater accuracy, if we expect to achieve any better sensitivity in the detection of velocity fields. This is the approach used at the Meudon Observatory. To localize a sunspot, we digitize the whole spectroheliogram (1151 by 1151 pixels, each pixel corresponding to the spatial resolution of the spectroheliogram) and apply an image-processing program to correct the solar image for geometric and photometric distorsions. This achieves a $15 \mathrm{~m} \mathrm{~s}^{-1}$ accuracy for each individual sunspot, and is limited only by the spatial resolution of the Meudon spectroheliograms (Mein and Ribes, 1990). We have computed the rotation rates of sunspots observed in summer (from April to October), from 1977 to 1983 , and have derived rotation rates from one year to the next.

Figure 1 shows the mean sunspot rotation, using a polynomial fit, averaged over the seven-year sequence from 1977 to 1983 , based on observations of 2900 sunspots. The mean synodic rotation laws for both hemispheres are

for the northern hemisphere: $\Omega=13^{\circ} 48-2.28 \sin ^{2} \theta+0.97 \sin ^{4} \theta$,

for the southern hemisphere: $\Omega=13^{\circ} .53-2^{\circ} .05 \sin ^{2} \theta-1.71 \sin ^{4} \theta$,

where $\theta$ is the latitude. As a few sunspots are observed at high latitudes, the rotation rate derived for these high-latitude belts is not significant. However, within a latitude range of $\pm 30^{\circ}$, the rotation rate derived from our data set is similar to that obtained by Howard et al. (1984).

There are noticeable differences in rotation rates from one year to the next. The mean rotation law for each year, showing the difference from the average rotation, is also given in Fig. 1. These departures appear as either a flattening or a steepening of the mean differential rotation profile. The observational effect was first discovered by Howard and LaBonte (1981) on the photospheric rotation rate, and has been referred to as a torsional oscillation. Their finding was interpreted by Ribes et al. (1985) and Ribes (1986), as a result of the Coriolis force which accelerates or decelerates the surface flow, in the vicinity of azimuthal convective rolls. The existence of azimuthal rolls has been inferred from a zonal meridional circulation pattern discovered by Ribes and Mein (1984). Such a pattern implies the existence of counter-rotating rolls, with boundary regions of upflows and downflows. These regions coincide with the large scale magnetic field pattern (Fig. 2) measured at Stanford (Hoeksema, 1984). In the present paper, we make use of the differences from two consecutive yearly rotation rates to reconstruct the azimuthal roll pattern, assuming that the change in the rotation from one year to the next is due to the Coriolis forces near the border of converging/diverging rolls. 


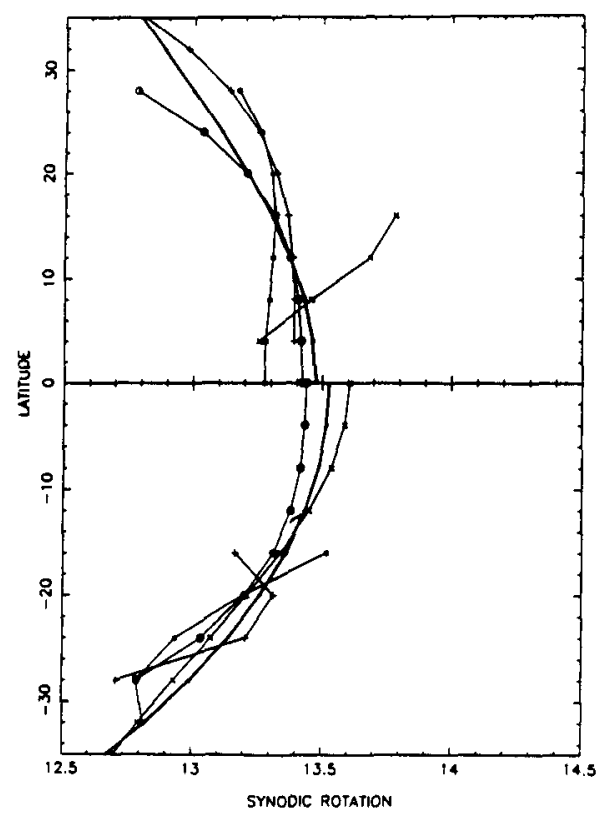

Fig. 1. The polynomial fits of rotation profiles derived from the digital analysis of 2900 sunspots from 1977 to 1983: solid thick curve refer to the mean differential rotation derived for the whole sample, while $+, *, 0$, and $\mathrm{x}$ refer to yearly rotation rate of 1977 , 1979, 1981 and 1983 respectively. Errors of the yearly means for $5^{\circ}$ latitude intervals are generally about $0.1^{\circ} /$ day (from $0.05^{\circ}$ to $0.2^{\circ}$ ).

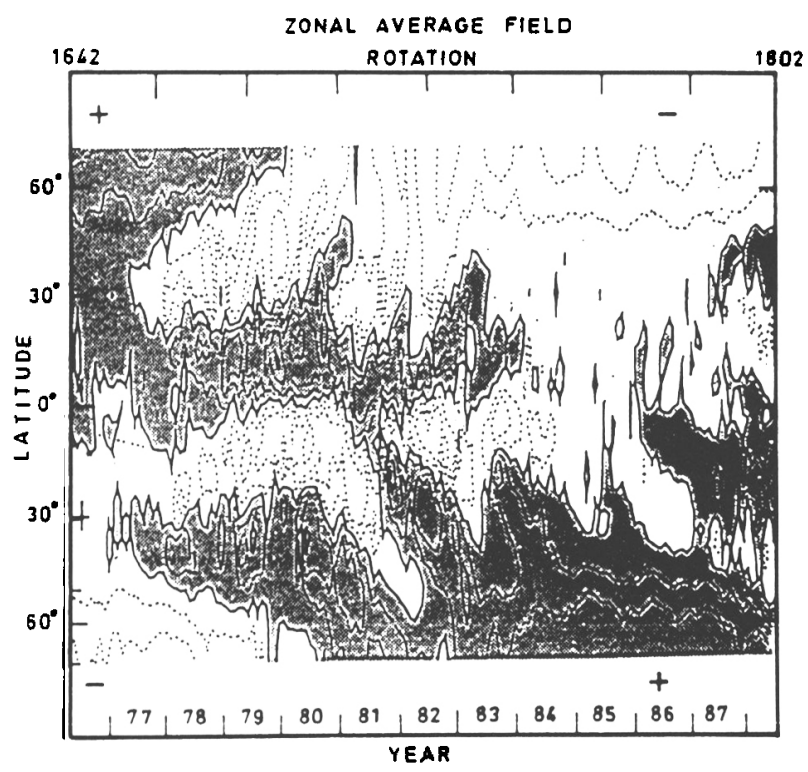

Fig. 2. Large-scale magnetic pattern measured at Stanford. The large-scale magnetic pattern coincides fairly well with the direct large-scale circulation of sunspots shown in Fig. 4. 


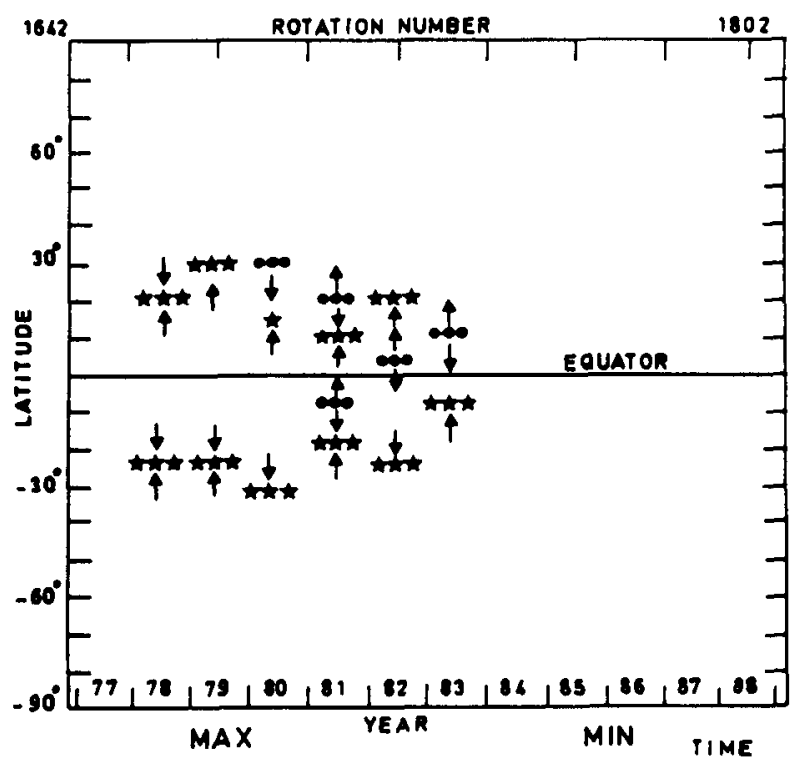

Fig. 3. Meridional circulation pattern deduced from the yearly changes in the differential rotation from one year to the next.

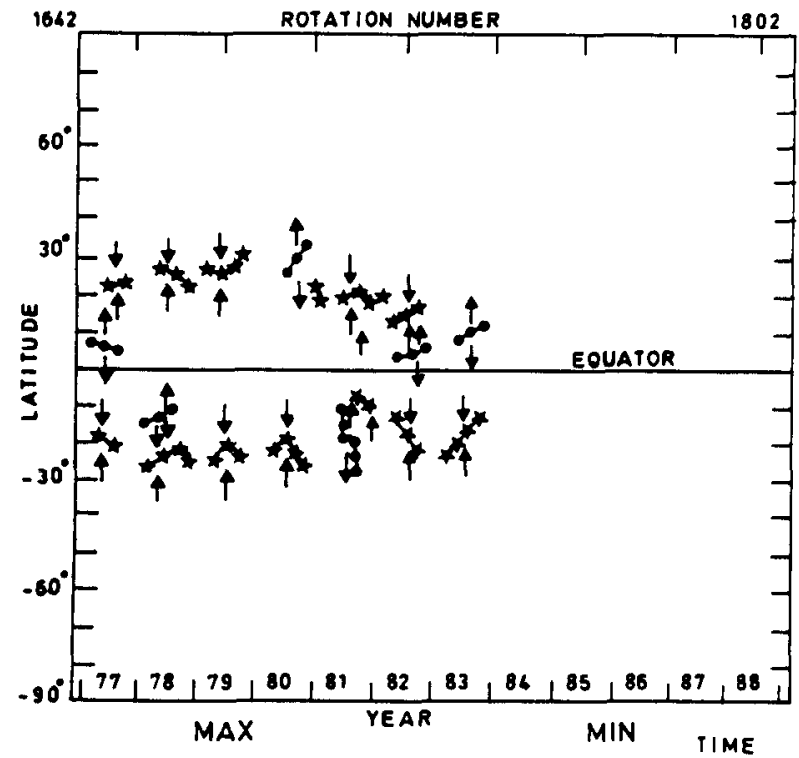

Fig. 4. Direct meridional circulation measurements (north-south drift of sunspots) for the period $1977-1983$. 
Results are shown in Fig. 3. On the other hand, the zonal meridional circulation (north-south motions) for the whole data set is a direct measurement (Ribes and Bonnefond, 1990), see Fig. 4. It is clear that the deformation of the mean rotation profile (flattening and steepening) can be explained by the presence of a pattern of converging rolls. Some discrepancies do exist, but these can be explained by the fact that our data cover half years rather than the whole years. The amplitude of the changes in the rotation rate obtained in our analysis is almost one order of magnitude greater than the amplitude of the torsional oscillation. A possible explanation for this is that sunspots probe deep into the convective layers while spectroscopic measurements refer to the photospheric plasma. It should also be pointed out that the accuracy of the rotation rate as determined by the a digital analysis of sunspots is significantly greater than by the Doppler measurements.

The poleward propagation of the rolls, as well as their direction of rotation, redistributes angular momentum at the surface and along the radius. This probably accounts for the rotation variations (two peaks per 11-year cycle) reported by Gilman and Howard (1984) on sunspots. Further comparison with Gilman and Howard's results will be published in a separate paper.

\section{References}

Balthasar, H., Vasquez, M., Wöhl, H.: 1986, Astron. Astrophys. 155, 87

Hoeksema, T.: 1984, thesis, Stanford University

Howard, R.H., Labonte, B.J.:1980, Astrophys. J. Letter 239, L33

Gilman, P.A., Howard, R.H.: 1984, Astrophys. J. 283, 385

Howard, R.H., Gilman, P.A., Gilman, P.I.: 1984, Astrophys. J. 284, 373

Mein, P., Ribes, E.: 1990, Astron. Astrophys. 227, 577

Ribes, E., Mein, P.: 1984, in Proc. Eur. Astron. Meeting, ed. R. Muller, Toulouse, p. 283

Ribes, E., Mein, P., Mangeney, A.: 1985, Nature 318, 170

Ribes, E., Bonnefond, F.: 1990, Geophys. Astrophys. Fluid Dyn., in press 